\section{Comparación del tamaño transversal de incisivos laterales maxilares sin microdoncia y con microdoncia en las maloclusiones}

\section{Comparison of the transverse size of maxillary lateral incisors without microdontia}

\section{Artículo Original}

\author{
Alejandra Olivia Acosta-Pelayo 1,a, Jaime Fabián \\ Gutiérrez-Rojo 1,b
}

${ }^{1}$ Universidad Autónoma de Nayarit, Facultad de Odontología, División de Estudios de Posgrado e Investigación,

Especialidad en Ortodoncia, Tepic, México.

${ }^{a}$ Cirujano Dentista.

${ }^{\mathrm{b}}$ Maestría en Salud Pública.

\section{Correspondencia:}

Alejandra Olivia Acosta-Pelayo: ale_oli91@hotmail.com Calle Josefa Ortiz de Domínguez no. 512 norte Colonia Santa Teresita, Tepic, Nayarit, México. Código postal 63020.

ORCID: 0000-0002-3033-1818

Coautor:

Jaime Fabián Gutiérrez-Rojo: jaimefg79@hotmail.com ORCID: 0000-0002-8795-096X

\section{Editora:}

Sandra Patricia Palomino-Gómez

Universidad Nacional Mayor de San Marcos. Lima, Perú.

Conflicto de interés: los autores declaran no tener conflictos de interés.

Fuente de financiamiento: autofinanciado.

\section{Resumen}

Objetivo. Determinar si existe una diferencia entre los tamaños transversales de los incisivos laterales maxilares en las diferentes maloclusiones de Angle y determinar la prevalencia de la microdoncia de incisivos laterales maxilares en las diferentes clases de Angle. Métodos. La investigación es de tipo descriptiva, transversal y observacional. El universo de estudio fueron 780 modelos de estudio pretratamiento de ortodoncia. La muestra fue de 190 modelos de estudio. Para determinar la presencia de microdoncia se utilizó el método de Binder y Cohen. Resultados. En la maloclusión de Clase I se encontró una frecuencia de microdoncia del 32\%, en la maloclusión Clase II fue del 35\% y en la maloclusión Clase III del 26\%. Los incisivos laterales superiores con microdoncia y sin microdoncia tienen una media estadísticamente diferente entre ellos a un nivel de confianza mayor al 95\%, esta diferencia se observa homogénea en cada una de las maloclusiones. Conclusiones. No se observaron diferencias estadísticamente significativas en el tamaño mesiodistal de los insicivos laterales maxilares al compararse entre las tres maloclusiones descritas por Angle. Se obtuvo mayor porcentaje de microdoncia en la maloclusión Clase II, y un menor porcentaje en la Clase III.

Palabras clave: Diente; Incisivo; Prevalencia; Maloclusión (fuente: DeCS BIREME).

\begin{abstract}
Objective. To determine if there is a difference between the transverse sizes of the maxillary lateral incisors in the different Angle malocclusions and to determine the prevalence of maxillary lateral incisors with microdontia in the different Angle classes. Methods. The research is descriptive, transversal and observational. The study universe was 780 orthodontic pre-treatment study models. The sample was 190 study models. Binder and Cohen's method was used to determine the presence of microdontia. Results. In Class I malocclusion a frequency of microdontia of $32 \%$ was found, in Class II malocclusion it was 35\% and in Class III malocclusion it was 26\%. The upper lateral incisors with microdontia and without microdontia have a statistically different mean between them at a confidence level greater than $95 \%$, this difference is observed homogeneously in
\end{abstract}

Recibido: 04/01/20

Aceptado: 20/03/20

Publicado: 04/08/20 
each of the malocclusions. Conclusions. No statistically significant differences in the mesiodistal size of the maxillary lateral incisors were observed when comparing the three malocclusions described by Angle. A higher percentage of microdontia was obtained in Class II malocclusion, and a lower percentage in Class III.

Keywords: Tooth; Incisor; Prevalence; Malocclusion (source: MeSH NLM).

\section{Introducción}

El "padre de la ortodoncia moderna", Eduard H. Angle ${ }^{1}$, postuló que en una oclusión normal existe una línea de oclusión curveada de manera uniforme y que además la cúspide mesiobucal del molar superior debe ocluir en el surco bucal del molar inferior. Con base en este postulado se clasificaron los tres tipos de maloclusión que son: Clase I, en la que las relaciones de los molares son ideales, pero la línea de oclusión no es correcta. La posición del molar inferior en la Clase II es en distal, mientras que en la Clase III en mesial de la relación molar ideal. Sin embargo, pudieran existir anomalías dentales que alteren una oclusión ideal.

Las anomalías dentales son malformaciones que se producen durante la etapa de morfodiferenciación por factores ambientales o alteraciones genéticas ${ }^{2}$, así como factores locales o sistémicos ${ }^{3}$. Estas aberraciones pueden ser de forma, número, tamaño, posición y color de los dientes ${ }^{4}$.

La microdoncia es una anomalía dentaria definida como la condición en la que los dientes son más pequeños del rango de variación normal ${ }^{2,5}$. Ésta es más frecuente en los incisivos laterales y los terceros molares ${ }^{3}$.

Shafer ${ }^{3}$ clasifica la microdoncia en tres tipos: microdoncia que afecta a un solo diente (puede estar afectada la raíz, la corona o ambas), microdoncia generalizada (dientes pequeños en mandíbulas grandes) y microdoncia generalizada verdadera (dientes con tamaño menor al normal). Ésta última se presenta en alteraciones sistémicas como enanismo hipofisiario o displasia espondiloepifisaria 5 .

Cuando se presenta microdoncia de incisivos laterales superiores se producen una serie de anomalías que a su vez originan maloclusiones al afectar las relaciones oclusales ${ }^{6}$. Las anomalías más frecuentes son: espacios interdentales, modificaciones en la forma y longitud del arco y migraciones dentales indeseables ${ }^{7}$. También se presenta la retención o erupción ectópica de los caninos, o su desplazamiento hacia palatino con la posible reabsorción radicular no deseable ${ }^{6}$. Estos cambios producen que se vea afectada la estética y que se puedan desencadenar problemas funcionales ${ }^{8,9}$.

El tipo de maloclusión que se genere por consecuencia de la presencia de incisivos laterales superiores con microdoncia puede variar en función del conjunto de todas las anomalías presentes en el aparato estomatognático. Además, conocer el tamaño mesiodistal de aquellos dientes con y sin microdoncia nos dará parámetros teóricos que ayuden a relacionar esta anomalía con cada una de las maloclusiones, por lo tanto es necesario determinar la cantidad de discrepancia entre el tamaño de los dientes al momento de realizar el diagnóstico, para lograr una buena intercuspidación y armonía entre los dientes superiores e inferiores, así como entre los dientes y la dimensión de la arcada para que se pueda dar una alineación y oclusión correctas ${ }^{10,11}$.

Algunos de los tratamientos que se pueden realizar son la colocación de restauraciones o prótesis. Se utiliza también el tratamiento de ortodoncia para la distribución de los espacios antes de la rehabilitación de dichas piezas ${ }^{9}$.

El método de Binder y Cohen, es un procedimiento para analizar y determinar la existencia de microdoncia del incisivo lateral superior, se basa en datos obtenidos por Moorrees y Moyers que mencionan que cuando se presenta entre un $12 \%$ y $14 \%$ más ancho el incisivo lateral superior que el incisivo lateral inferior, se considera que no hay microdoncia ${ }^{7}$. En este método se deben hacer coincidir el incisivo lateral superior e inferior; para que no exista microdoncia es necesario que el incisivo lateral superior sea $0,7 \mathrm{~mm}$ más ancho mesiodistalmente que el incisivo lateral inferior. El método de Binder y Cohen ha sido utilizado por investigadores como Gómez Fernández et al ${ }^{7}$ y Gutiérrez Rojo $e t a l^{6}$ para realizar investigaciones en las cuales se determinó la prevalencia de microdoncia del incisivo lateral superior.

Investigaciones realizadas en Turquía de Aren et al. ${ }^{2}$, en Colombia de Bedoya-Rodríguez et al. ${ }^{4}$ y Yemen por Aldhorae et al. ${ }^{8}$ otorgan datos sobre la prevalencia y el manejo de las anomalías dentales; una de las anomalías más frecuentes es la microdoncia del incisivo lateral superior ${ }^{3}$. Sin embargo, en el presente estudio se compara no sólo la frecuencia, sino también el tamaño transversal del incisivo lateral superior en aquellos dientes que presentan o no microdoncia y en cada una de las maloclusiones brindando información relevante para el diagnóstico y tratamiento, también proporciona una base para futuras investigaciones, abonando así, en el conocimiento sobre el tema.

Por lo tanto, los objetivos de este estudio son determinar si existe una diferencia entre los tamaños transversales de los incisivos laterales maxilares en las diferentes maloclusiones de Angle y determinar la prevalencia de la microdoncia de incisivos laterales maxilares en las diferentes clases de Angle.

\section{Métodos}

La investigación fue de tipo descriptiva, transversal y observacional. El universo de estudio fueron 780 modelos 
de estudio pretratamiento de ortodoncia de la Especialidad de Ortodoncia de la Universidad Autónoma de Nayarit del año 2015 al 2019. El tamaño de muestra se calculó con un nivel de confianza del $95 \%$, un error del $3 \%$ y una proporción del 5\%. Como resultado el tamaño de muestra fue de 190 modelos de estudio. Se utilizó un muestreo consecutivo de los modelos que cumplieron con los criterios de inclusión.

Se realizó la evaluación pertinente y se dio el consentimiento con dictamen aprobatorio correspondiente por parte del comité de ética de la Universidad Autónoma de Nayarit, con el número de registro CEIUAO-02-2020.

Los criterios de inclusión fueron: modelos de estudio con los dientes permanentes completamente erupcionados (los terceros molares no fueron considerados), y que las impresiones fueran hechas en el mismo gabinete de estudios. Se excluyeron: los modelos de estudio que presentaran fracturas, cavidades, desgastes o restauraciones que afectaran los contactos interproximales o la anatomía dental.

Los materiales que se utilizaron fueron: los modelos de estudio, un calibrador digital marca Mitutoyo, pinceles de brocha fina, hojas de recolección de datos, lapicera, corrector y una computadora.

Para determinar la presencia de microdoncia se utilizó el método de Binder y Cohen, el cual utiliza los incisivos laterales del maxilar y mandíbula. Se procedió a retirar cualquier partícula que interfiriera en la medición con los pinceles de brocha fina y se midió al ancho mesiodistal de los incisivos laterales superiores e inferiores con el calibrador digital marca Mitutoyo. La totalidad de las mediciones en los modelos fue realizada por un solo investigador, y los datos fueron recolectados en hojas de registro, se realizó el índice de correlación interclase (ICC) para la evaluación intraoperador. Se tabuló en el programa Microsoft Office Excel 2007. El análisis de los datos se realizó en el programa Statistical Package for the Social Sciences (SPSS) versión 18 por medio de la estadística descriptiva, la normalidad de los datos (utilizando la prueba de Shapiro-Wilk) y las pruebas de t de Student y ANOVA. Se trabajó a un nivel de significancia del 5\%.

\section{Resultados}

Se obtuvo 0,902 en la prueba de correlación interclase (ICC) para la evaluación intraoperador.

En la maloclusión de Clase I se encontró una frecuencia de microdoncia del $32 \%$, de los dientes con microdoncia el 58\% fue bilateral, el 33\% fue unilateral del lado derecho y el $9 \%$ fue unilateral del lado izquierdo. El promedio transversal de los incisivos laterales maxilares sin microdoncia fue de 7,43 $\pm 0,53 \mathrm{~mm}$ y de los incisivos laterales maxilares con microdoncia de 6,68 \pm 0,56 $\mathrm{mm}$. El resto de la estadística descriptiva se encuentra en la tabla 1 .

La maloclusión de Clase II presentó una frecuencia de microdoncia del 35\%. En los dientes con microdoncia en esta maloclusión se encontró que el $46 \%$ era bilateral, el 19\% fue unilateral del lado derecho y el 35\% unilateral del lado izquierdo. El tamaño de los incisivos laterales maxilares sin microdoncia presentó un promedio de 7,37 $\pm 0,56 \mathrm{~mm}$, los incisivos laterales maxilares con microdoncia presentaron un promedio de 6,61 \pm $0,59 \mathrm{~mm}$ (Tabla 1).

Para la maloclusión de Clase III la prevalencia de microdoncia fue del 26\%. Se encontró que el $43 \%$ fue bilateral, el 28,5\% fue unilateral del lado derecho y el $28,5 \%$ unilateral del lado izquierdo. Los incisivos laterales maxilares sin microdoncia presentaron un promedio mesiodistal de 7,52 $\pm 0,43 \mathrm{~mm}$ y los incisivos laterales maxilares que presentaron microdoncia el promedio fue de $6,45 \pm 0,59 \mathrm{~mm}$ (Tabla 1$)$.

Se incluye el número de modelos seleccionados que presentan microdoncia del incisivo lateral superior $y$ aquellos que no presentan en cada tipo de maloclusión (Tabla 2).

Se encontraron diferencias estadísticas significativas con la prueba $\mathrm{t}$ de Student entre los incisivos laterales

Tabla 1. Medidas mesiodistales de los incisivos laterales maxilares

\begin{tabular}{|c|c|c|c|c|c|c|}
\hline & \multicolumn{3}{|c|}{ Microdoncia } & \multicolumn{3}{|c|}{ Sin Microdoncia } \\
\hline & Clase I & Clase II & Clase III & Clase I & Clase II & Clase III \\
\hline Promedio & 6,68 & 6,61 & 6,45 & 7,43 & 7,37 & 7,52 \\
\hline Desviación Estándar & 0,56 & 0,59 & 0,59 & 0,53 & 0,56 & 0,43 \\
\hline Máximo & 7,8 & 7,6 & 7,6 & 8,5 & 8,6 & 8,4 \\
\hline Mínimo & 5,4 & 5,04 & 5,7 & 6,3 & 6,3 & 6,7 \\
\hline Rango & 2,4 & 2,56 & 1,9 & 2,2 & 2,3 & 1,7 \\
\hline
\end{tabular}

Tabla 2. Número de modelos seleccionados por maloclusión con y sin microdoncia de incisivos laterales maxilares

\begin{tabular}{ccc}
\hline Maloclusión & Sin microdoncia & Microdoncia \\
\hline I & 43 & 20 \\
II & 41 & 23 \\
II & 46 & 17 \\
\hline
\end{tabular}


maxilares sin microdoncia comparados con los incisivos laterales maxilares con microdoncia en cada una de las maloclusiones (Tabla 3).

Se comparó el tamaño mesiodistal de los incisivos laterales maxilares con microdoncia entre los tres tipos de maloclusiones, mediante la prueba de ANOVA, no encontrándose diferencias estadísticamente significativas $(\mathrm{p}=0,619)$.

\section{Discusión}

Algunos estudios sugieren que los pacientes con maloclusión Clase III presentan un ancho mesiodistal más pequeño, sin embargo, en otros estudios no hay diferencias significativas entre las diferentes maloclusiones y los incisivos laterales superiores con microdoncia, el presente artículo brinda información actual y relevante al respecto ${ }^{10}$.

Autores como Binder y Cohen ${ }^{10}$ y Hunter ${ }^{12}$ han desarrollado procedimientos para medir el ancho mesiodistal de los dientes. Estas medidas son indispensables para interceptar el desarrollo de irregularidades faciales y oclusales, la posibilidad de que existan discrepancias en la oclusión por cambios en la intercuspidación durante el tratamiento de ortodoncia y posterior a este, e identificar aquellos dientes con microdoncia en las maloclusiones leves ${ }^{12}$.

Gómez Fernández et $a l^{7}$ utilizaron el método de Binder y Cohen para determinar si existe o no microdoncia del incisivo lateral superior, y lo describen como un método sencillo, práctico y rápido. Se obtuvieron como resultados de su estudio que el $42,5 \%$ de los modelos en los cuales utilizaron el método de Binder y Cohen presentaron microdoncia de los incisivos laterales superiores. En este estudio se obtuvo menor frecuencia de microdoncia del incisivo lateral superior en cada una de las maloclusiones, en la Clase I se obtuvo una frecuencia del $32 \%$, en la maloclusión Clase II fue del $35 \%$ y en la Clase III del $26 \%$.

Aren $e t a l .{ }^{2}$ realizaron un estudio en Turquía y concluyeron que la prevalencia de microdoncia y forma de clavija de los incisivos laterales en el maxilar fue del 0,54, mientras que Singh ${ }^{3}$ reportó que la microdoncia presenta en China una prevalencia del $2,58 \%$ en su mayoría en los incisivos laterales, sin embargo, con dicha prevalencia es mayor en este estudio.

En un estudio realizado por Aldhorae et al. ${ }^{8}$ determinaron que el incisivo lateral superior izquierdo fue el diente que presentó más frecuentemente microdoncia con un porcentaje del 3,91\%, en la presente investigación el porcentaje de microdoncia del incisivo lateral izquierdo superior en la maloclusión Clase III y II fue mayor, sin embargo, en la Clase I el porcentaje fue menor comparado con dicho estudio.

Gutiérrez-Rojo et al. ${ }^{6}$ mediante el método de Binder y Cohen determinaron que la Clase III presentó el menor porcentaje de dientes con microdoncia al igual que en este artículo; sin embargo, Gutiérrez-Rojo et al. obtuvieron un porcentaje del $4,4 \%$, mientras que en esta investigación se determinó un porcentaje de $26 \%$ de microdoncia en la clase III. En ambos artículos se encontró que la Clase II presenta el mayor porcentaje de dientes con microdoncia. Los dos artículos concuerdan que el menor tamaño mesiodistal de los incisivos laterales maxilares con microdoncia se presentó en la maloclusión Clase III.

Gómez-Fernández et al. ${ }^{11}$ compararon el tamaño de los incisivos laterales con y sin microdoncia en las diferentes maloclusiones y no encontraron diferencias estadísticas en la maloclusión de clase III, a diferencia de este estudio en el que se encontraron diferencias significativas en las tres maloclusiones. En ambas investigaciones la mayor prevalencia de microdoncia del incisivo lateral maxilar se encontró en la maloclusión Clase II, sin embargo, Gómez- Fernández et al. ${ }^{11}$ encontraron prevalencias de $7,95 \%$ en la Clase I, 12,38\% en la maloclusión II y $4,4 \%$ en la maloclusión Clase III. En este estudio se encontraron porcentajes mayores en los tres tipos de maloclusiones, con una frecuencia de microdoncia del $32 \%$ en la maloclusión Clase I, del $35 \%$ en la maloclusión Clase II y en la maloclusión Clase III del 26\%. Gómez- Fernández et al. ${ }^{11}$ determinaron la prevalencia de incisivo lateral superior con microdoncia de 40,5\%.

Fekonja ${ }^{13}$ desarrolló una investigación que dio como resultado que la microdoncia constituía el 15,2\% del total de anomalías dentales, y de los dientes que presentaban microdoncia el $67,9 \%$ afectaba a los incisivos laterales superiores, mientras que Kwang et al. ${ }^{14}$ en un estudio realizado en gemelos de Corea encontraron que el porcentaje de microdoncia es del 3\%, dicho porcentaje fue mayor en este estudio en las maloclusiones Clase II y III.

Para llevar a cabo el método de medición en el presente estudio se siguió la metodología de las investigaciones realizadas por Fekonja ${ }^{13}$ en Slovenia, en la cual se realizaron mediciones del ancho mesiodital de incisivos laterales superiores en modelos de yeso utilizando un vernier digital para determinar la prevalencia de algunas anomalías, en

Tabla 3. Resultados de la prueba de t de Student entre los incisivos laterales maxilares sin anomalías de tamaño con los incisivos laterales con microdoncia

\begin{tabular}{cccc}
\hline & Clase I & Clase II & Clase III \\
\hline $\mathrm{t}$ & $-5,57$ & $-9,97$ & $-5,9$ \\
$\mathrm{p}$ & $0,001^{* *}$ & $0,001^{* *}$ & $0,001^{* *}$ \\
\hline
\end{tabular}

**significativo $\mathrm{p}<0,01$ 
donde todas las mediciones fueron realizadas por un mismo examinador para evitar la discrepancia de criterios y el sesgo en la medición.

Este estudio es importante para la práctica clínica ya que identifica el tamaño mesiodistal de los incisivos laterales maxilares en cada una de las maloclusiones, este dato tiene relevancia por la influencia que ejercen estos dientes en el arco dental. Conociendo el tamaño de los incisivos laterales maxilares se puede determinar desde el inicio del tratamiento los resultados que se esperan obtener, ya que si existe microdoncia del incisivo lateral superior se desencadenan anomalías que producen maloclusiones; por lo tanto, tener valores del ancho mesiodistal en cada una de las maloclusiones es un conocimiento teórico que se puede aplicar a la práctica clínica durante las tres fases del tratamiento de ortodoncia.

Una de las limitaciones de este estudio es que los modelos de estudio de la muestra para la obtención de los datos son de pacientes que acudieron a tratamiento de ortodoncia al Posgrado de Ortodoncia de la Universidad Autónoma de Nayarit, lo que genera una limitación en la extrapolación de los resultados a la población en general. Se recomienda ampliar la muestra por regiones geográficas en México, agregar más variables que se relacionen con la microdoncia del incisivo lateral maxilar como la comparación de la microdoncia con la forma de arco, la diferencia entre sexos y la genética, quedando abiertas estas líneas del conocimiento para futuras investigaciones.

Se concluye que no se observaron diferencias estadísticamente significativas en el tamaño mesiodistal de los incisivos laterales maxilares al compararse entre las tres maloclusiones descritas por Angle. En la presente investigación se obtuvo mayor porcentaje de microdoncia en la maloclusión Clase II, y un menor porcentaje en la Clase III.

\section{Referencias bibliográficas}

1. Proffit W, Fields H, Sarver D. Ortodoncia contemporánea. Quinta Edición. Barcelona: Editorial Elsevier; 2014.p.2-3.

2. Aren G, Güven Y, Tolgay CG, Özcan İ, Bayar ÖF, Köse TE, et al. The Prevalence of Dental Anomalies in a Turkish Population. J Istanb Univ Fac Dent. 2015 Aug [citado 2019 Oct 13];49(3):23-8. Disponible en: https:// search.ebscohost.com/login. aspx?direct $=$ true $\& \mathrm{db}=\mathrm{d}$ dh\&AN $=110907587 \&$ site $=$ ehost-live

3. Singh B. Management of Localized Microdontia of Maxillary First Premolar - A Case Report Clin Dent (0974-3979) [Internet]. 2017 Jun [citado 2019 Oct 13];11(6):21-5. Disponible en: https:// search.ebscohost.com/login.aspx? direct $=$ true $\& \mathrm{db}=\mathrm{d}-$ dh\&AN=125364944\&site=ehost-live

4. Bedoya-Rodríguez A, Collo-Quevedo L, Gordillo-Meléndez L, Yusti-Salazar A, Tamayo-Cardona JA, Pérez-Jaramillo A, et al. Anomalías dentales en pacientes de ortodoncia de la ciudad de Cali, Colombia. CES Odontol [Internet]. 2014 Jan [citado 2019 Oct 13];27(1):45-54. Disponible en: https://search.ebscohost.com/login.aspx?direct=true $\& \mathrm{db}=\mathrm{ddh} \& \mathrm{AN}=98628410 \&$ site $=$ ehost-live
5. Singhal A, Singhal P, Gupta R, Jarial KD. True Generalized Microdontia and Hypodontia with Spondyloepiphyseal Dysplasia. Case Rep Dent [Internet]. 2013 Jan [citado 2019 Oct 30];1-4. Disponible en: https:// search.ebscohost.com/login. aspx?direct $=$ true $\& \mathrm{db}=\mathrm{d}$ dh\&AN=94854799\&site=ehost-live

6. Gutiérrez-Rojo MF, Gutiérrez-Rojo JF, Gallardo-Espinoza E, Lomeli-Armenta A, Gutiérrez-Villaseñor J, Rojas-García AR. Microdoncia de incisivos laterales maxilares en maloclusiones dentales. Rev Tamé. 2017;6(16):565-568.

7. Gómez-Fernández D, Rivas-Gutiérrez R, Gutiérrez-Rojo JF. Prevalence of upper lateral incisor microdontia in a mexican population. CES Odontol [Internet]. 2013 Jul [citado 2019 Oct 13];26(2):67-73. Disponiblle en: https://search.ebscohost.com/login.aspx?direct=true\&d$\mathrm{b}=$ ddh \&AN=94592533\&site=ehost-live

8. Aldhorae KA, Altawili ZM, Assiry A, Alqadasi B, Al-Jawfi KA, Hwaiti H. Prevalence and Distribution of Dental Anomalies among a Sample of Orthodontic and Non-orthodontic Patients: A Retrospective Study. J Int Oral Health [Internet]. 2019 Sep [citado 2019 Dec 14];11(5):309-17. Disponiblle en: https:// search.ebscohost.com/login. aspx?direct $=$ true $\& \mathrm{db}=\mathrm{d}$ dh\&AN=138880128\&site=ehost-live

9. Tirone F, Salzano S, Rolando E. Adhesive esthetic treatment of non-syndromic maxillary anterior microdontia in young high-demanding patients: a case series. J Esthet Dent. [Internet]. 2016 Winter [citado 2019 Oct 13];11(4):520-37. Disponible en: https:// search.ebscohost.com/login.aspx?direct $=$ true $\& \mathrm{db}=\mathrm{d}$ dh\&AN=120323242\&site=ehost-liv

10. D La Torre C, Gurrola B, Casasa A. Manejo miltidiciplinario en pacientes con laterals superiors microdónticos. Caso clínico. Multidisciplinary approach in patient with upper lateral incisor microdontia. Case report Rev Mex Ortodon; 2016;4(2): 136-141.

11. Gómez-Fernández D, Rivas-Gutiérrez R, Gutiérrez-Rojo JF. Prevalence of upper lateral incisor microdontia in a mexican population. CES Odontol [Internet]. 2013 Jul [citado 2019 Dec 15];26(2):67-73. Disponible en: https://search.ebscohost.com/login.aspx?direct=true\&d$\mathrm{b}=\mathrm{ddh} \& \mathrm{AN}=94592533 \&$ site $=$ ehost-live

12. Kubodera T, Zárate C, Lara E, Montiel N, Esquivel G, Centeno C. Dimensiones coronales mesiodistales en la dentición permanente de mexicanos. Rev ADM 2008; LXV (3):141-149.

13. Fekonja A. Prevalence of dental developmental anomalies of permanent teeth in children and their influence on esthetics. J Esthet Restor Dent. [Internet]. 2017 Jul 8 [citado 2019 Dec 15];29(4):276-83. Disponible en: https://search.ebscohost.com/login.aspx?direct=true $\& \mathrm{~d}$ $\mathrm{b}=\mathrm{ddh} \& \mathrm{AN}=124586775 \&$ site $=$ ehost-live

14. Kwang Ho Jeong, Daeeun Kim, Yun-Mi Song, Joohon Sung, Young Ho Kim. Epidemiology and genetics of hypodontia and microdontia: A study of twin families. Angle Orthod. [Internet]. 2015 Nov [citado 2019 Dec 14];85(6):980-5. Disponible en: https:// search.ebscohost. com/login. aspx?direct $=$ true $\& \mathrm{db}=\mathrm{d}$ dh\&AN=111182928\&site=ehost-live 
